\section{Nie waren sie so wichtig wie heute}

\author{
Es ist kein Zufall, dass trotz der viel versprechenden deutschen Nachhaltig- \\ keitsstrategie das Konzept Nachhaltigkeit seit der Verkündung der Agenda \\ 2010 nicht mehr auf der Tagesordnung der Classe Politique steht. Das \\ gegenwärtige Versagen der Politik in Sachen Nachhaltigkeit klar aufzuzeigen \\ ist eine der wesentlichen Funktionen von Nachhaltigkeitsindikatoren.
}

$\mathrm{N}$ Von Joachim Spangenberg gänzung zum gegenwärtig dominierenden Credo vom Primat der Ökonomie. Gerade deshalb ist es notwendig, an die multiplen Optimierungsziele des Konzepts zu erinnern, das ja offiziell noch immer Geschäftsgrundlage der deutschen und europäischen Politik ist. Um diese Kritik wirkungsvoll formulieren und transportieren zu können, müssen die benutzten Indikatoren die komplexen Zielsetzungen der Nachhaltigkeit ebenso widerspiegeln wie die dynamische Interaktion der Teilaspekte. Gleichzeitig müssen sie nachvollziehbar sein und dürfen eine überschaubare Zahl nicht überschreiten. Die Quadratur des Kreises scheint dagegen eine vergleichsweise einfache Aufgabe. Um sie zu lösen müssen das Gesamtkonzept der Nachhaltigkeit systematisch strukturiert, Indikatoren transparent selektiert und verständliche Darstellungsformen gefunden werden.

\section{> Nachhaltigkeit}

An jeder menschlichen Handlung sind alle vier Dimensionen in wechselnder Gewichtung beteiligt; oft sind die Grenzen zwischen ihnen nur mühsam zu erkennen. Die Trennung ist also künstlich, aber insofern sinnvoll, als die beschriebenen Komponenten der Gesamtheit von ,,menschlicher Gesellschaft und ihrer Umwelt" über verschiedene, nicht vergleichbare Funktionslogiken und um Größenordnungen unterschiedliche Zeitstrukturen verfügen. Schwache Vergleichbarkeit und fehlende Kommensurabilität bedeuten auch, dass es nicht möglich ist, Situationscharakteristika aller vier Dimensionen zu einem aussagefähigen, in monetären oder physischen Einheiten gemessenen ,Nachhaltigkeitsindex“ zu aggregieren. Damit entfallen auch Bewertungsverfahren wie Kosten-Nutzen-Analysen, die auf eine derartige Aggregation angewiesen sind (2). Entscheidungen zur Nachhaltigkeit bauen eher auf die prozedurale Rationalität von Multikriterienund Positionsanalysen (siehe Tabelle 1).
Nachhaltige Entwicklung umfasst ökologische, soziale, institutionelle und ökonomische Dimensionen, bei Ökonomen auch Natur-, Human-, So- zial- und Sachkapital genannt (1). Es handelt sich weder um die „Umweltpolitik für das 21. Jahrhundert", noch um die einfache Addition unterschiedlicher Politikbereiche. Es handelt sich um die weitaus anspruchsvollere Zielsetzung, ökologische Toleranzkriterien mit expliziten sozialen, institutionellen und ökonomischen Nachhaltigkeitszielen zu einem Gesamtkonzept zu verbinden, wobei der Stellenwert der einzelnen Ziele durchaus umstritten ist.

Nachhaltigkeit ist in dieser Sicht ein dynamisches Konzept; nachhaltige Entwicklung ist ein Prozess der Koevolution die vier genannten Dimensionen oder Subsysteme. Wie bei jedem Evolutionsprozess handelt es sich um dynamische Musterbildungen fern des Gleichgewichts, der ungerichtete Lernprozesse des Systems und gerichtete der ökonomischen Akteure zugrunde liegen. Diese sind mit den Mitteln der neoklassischen Ökonomie nicht angemessen zu erfassen; deren Apparat ist demnach nicht in der Lage, die für die Nachhaltigkeit der wirtschaftlichen Entwicklung maßgeblichen Eigenschaften zu bestimmen. Insbesondere ist zwischen den Eigenschaften zu unterscheiden, die für die Nachhaltigkeit der Einzelsysteme/Dimensionen gemäß ihrer eige-

nen Reproduktionslogik entscheidend sind (reflexive Kernkriterien, Core), und denen, die eine nachhaltigkeitsgerechte Interaktion der Systeme sicherstellen (Integrationsfelder, Linkages).

$\mathrm{Zu}$ allen diesen Kriterien sind Indikatoren zu definieren, ohne die Aufnahmefähigkeit der Adressaten/innen zu überfordern. Hierzu dient das Prisma der Nachhaltigkeit, eine graphische Darstellung der vier Dimensionen als gleichseitiger Tetraeder mit den sechs Integrationsfeldern als Kanten. Es handelt sich dabei um ein heuristisches Mittel zur Kommunikation von Komplexität, mit dessen Hilfe versucht wird, durch Strukturierung Verständlichkeit zu erzielen. Befragungen von Nutzern/innen des Prismas haben ergeben, dass

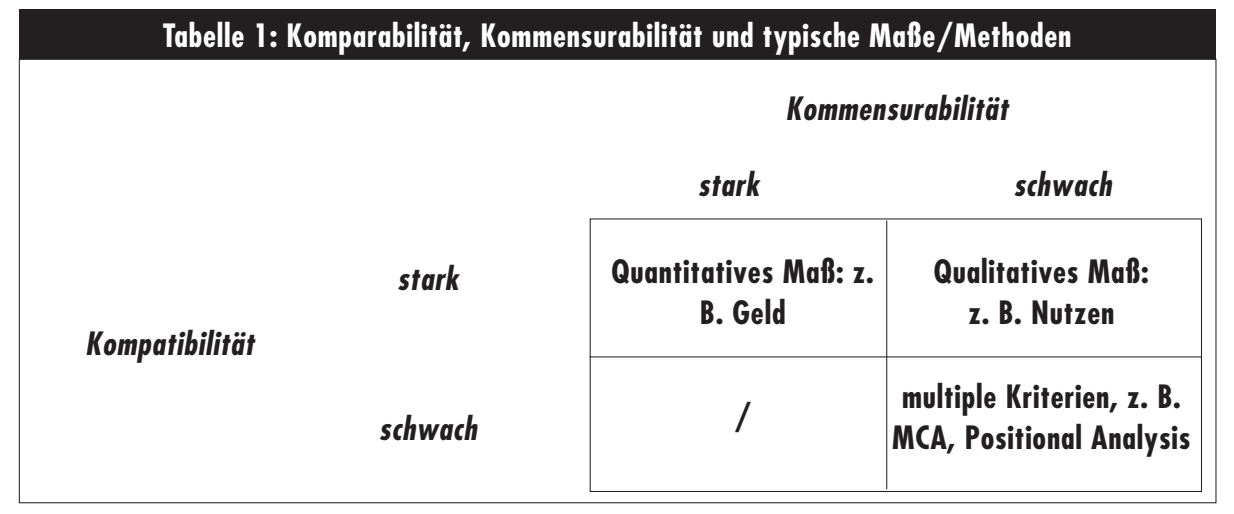

Quelle: eigene Darstellung

sie sich mit seiner Hilfe in der Lage fühlten, 20 bis 30 Indikatoren gleichzeitig wahrzunehmen. Dabei sind die Integrationsfelder von besonderer Bedeutung: für jede der Dimensionen lassen sich leicht gesellschaftliche Akteure ausmachen, deren Interessen in diesen Bereichen liegen, und die von ihnen in der politischen Debatte entsprechend vertreten werden. Da Nachhaltigkeit jedoch Integration bedeutet, die ausgleichende Wirkung diskursiver Prozesse notwendig, also die politische Gestaltung der Integrationsfelder.

\section{Indikatoren}

Am besten untersucht ist bisher die Umwelt und ihre Berücksichtigung in ökonomischen Prozessen. Das betrifft Quellen und Rollen quantitativer und qualitativer Belastungen sowie die Risiken, die unterschiedliche Landnutzungsmuster für die biologische Vielfalt darstellen. Es ist recht offensichtlich, dass die vorrangigen ökologischen Ziele einer Nachhaltigkeitspolitik in den Industriestaaten sein müssen den Druck auf die ökologischen Systeme zu verringern und Regenerationskapazitäten schaffen.

Daraus ergeben sich für die ökologische Dimension unmittelbar drei Indikatoren: Ressourcenver- 
brauch oder Umweltraumnutzung, Toxizitätsindex, und Größe der Naturschutzflächen. Da ein Toxizitätsindex auf europäischer Ebene noch in Bearbeitung ist, und da mit verringerten Gesamtstoffströmen bei einer geeigneten Chemiepolitik auch das Volumen der toxischen Substanzen abnimmt, wird dieser bei der Auswahl von insgesamt 20 Indikatoren zurückgestellt. Als Interlinkages sind damit Ressourcenintensität oder -produktivität (ökologisch-ökonomisch), Umweltkrankheiten (öko-sozial, als Ergänzung zur Ökotoxizität) und Regelungen des Umweltrechts (ökologisch-institutionell) plausibel. Alternativ könnten auch Ressourcenpreise als ökologisch-ökonomischer Indikator oder Umweltnutzungsmuster (nachhaltiger Konsum, sozial-ökologisch) als Indikatoren ausgewählt werden, wenn dies den akuten Problemen im jeweiligen Land besser entspräche.

Besondere Probleme aufgrund mangelnder Vorarbeiten bereitet die ökonomische Dimension, umfassend verstanden als Bestand, Herstellung, Austausch und Nutzung von Gütern und Dienstleistungen in Erwerbs- und Nichterwerbswirtschaft, einschließlich ihrer Mittel und Resultate wie Geld, Gewinne, Vermögen, Akkumulation, etc.. Die Nachhaltigkeit der Wirtschaft impliziert eine dynamische Stabilität, die weder Verharren in lock-in's noch Volatilität und hohe Krisenanfälligkeit toleriert, und die zugleich eine auf Nachhaltigkeit ausgerichtete Entwicklung in realer Zeit, also pfadabhängig und irreversibel ist. System-Charakteristika, die das bewirken, sind insbesondere Sensitivität, Beharrungsvermögen, Resilienz und Adaptivität sowie die Fähigkeit, abwägend die Integrität der sozio-ökonomischen wie natürlichen Umgebungssysteme zu gewährleisten. Strukturelle Voraussetzungen dieser Eigenschaften sind Diversität, Redundanz und die Dominanz negativer Rückkopplungsmechanismen, sowie Mechanismen, die die „Bedürfnisse“ der Parallelsysteme als Restriktionen für die Wirtschaft fühlbar machen. Für alle diese Charakteristika fehlen erprobte Indikatoren (3).

Diese Vorgehensweise systematischer Ableitung plus politischer Prioritätensetzung kann schrittweise weiter geführt werden und ermöglicht es, zum Beispiel die oft benutzten Strukturindikatoren der EU auf ihre Eignung als Nachhaltigkeitsindikatoren zu überprüfen. Diese weisen, so wie sie unter der irischen Präsidentschaft im Frühjahr 2004 beschlossen wurden, deutliche Schwächen auf, die in einem zweistufigen Prozess unter anderem mit Hilfe der oben gennanten Überlegungen behoben werden könnten.

\section{DIMENSIONEN}

\section{Wirtschaftsindikatoren}

- Wirtschaftliche Entwicklung (BIP)

- Inflationsrate

\section{Sozialindikatoren}

- Armut (HPI 2, Armutsindex für Industriestaaten des Entwicklungsprogramms der Vereinten Nationen)

- Lebenserwartung

\section{Umweltindikatoren}

- Umweltraum-Ausnutzung (Energie, Material, Land)

- Naturschutzfläche (Schutzstandard nach IUCN Klasse 3 bis 5)

\section{Institutionelle Indikatoren \\ - Wahlbeteiligung \\ - Pluralismus der Medien}

INTEGRATIONSFELDER

Ökologisch-ökonomische Indikatoren

- Ressourcenproduktivität (GDP/TMI)

- Zusammensetzung des Steueraufkommens aus der Belastung von Umwelt, Arbeit und Kapital

\section{Sozial-ökonomische Indikatoren}

- Einkommensverteilung

- Arbeitslosenquote

Sozial-ökologische Indikatoren

- Umweltbedingte Gesundheitsprobleme

- Zugang zu öffentlichen Gütern

Ökonomisch-institutionelle Indikatoren

- Korruptionshäufigkeit (Transparency International Index)

- Verbreitung von Arbeitnehmermitbestimmung

Sozial-institutionelle Indikatoren

- Geschlechtergerechtigkeit

- Verlässliche ausreichende Stützung durch das soziale Sicherheitssystem

Ökologisch-institutionelle Indikatoren

- Verbandsklagerecht für Umweltorganisationen

- Akteneinsichtsrechte zu Umweltbelangen

In einem „Post-Dublin-Prozeß“ könnten zunächst dringende technische Verbesserungen innerhalb der bestehenden Struktur vorgenommen werden. Damit wären einige Verbesserungen des Systems erreicht, erhalten blieben aber die einseitige Aufteilung der Themenfelder und die mangelnde Integration der Dimensionen. Um diese zu überwinden wäre ein zweiter, ein mittel- und langfristiger „Beyond-DublinProzeß" notwendig. In diesem sollte die mehr historisch denn sachlich begründete Gliederung der Strukturindikatoren durch eine systematische und aus dem Konzept Nachhaltigkeit abgeleitete Struktur ersetzt werden.

Wählt man jeweils nur zwei Schlüsselindikatoren, so ergibt sich, nach Dimensionen und Integrationsfeldern geordnet, ein Satz von zwanzig Indika- toren, der auf Grund der systematischen Herleitung alle Felder gleichmäßig abdeckt (siehe Kasten rechts).

Dieser oder ein ähnlicher Indikatorensatz wäre eine langfristig geeignete Grundlage, um den Fortschritt der nachhaltigen Entwicklung in Europa wie national zu verfolgen.

\section{Fazit}

Die Operationalisierung von Nachhaltigkeit mittels Indikatoren ist dann möglich, wenn sie nicht als statische, normativ formulierte Zielsetzungen verstanden werden, sondern als Bestandteile und Ergebnisse eines dynamischen, gerichteten aber ergebnisoffenen Prozesses. Sie sind sogar notwendig, um Fehlleistungen zu identifizieren, Rückkoppelungen zu ermöglichen und Handlungsbedarf zu formulieren.

Zielverfehlungen erkennbar machen heißt auch, aus vagem Unbehagen konkrete Kritik machen, also allgemeine Unzufriedenheit politikfähig zu machen. Insofern sind Nachhaltigkeitsindikatoren keine akademische Fingerübung, sondern ein wesentlicher Beitrag zum gesellschaftspolitischen Diskurs (4).

\section{Anmerkungen}

(1) Serageldin, I.: Expanding the Measure of Wealth. Indicators of Environmentally Sustainable Development. World Bank, Washington, D.C. 1997.

(2) Söderbaum, P.: Ecological Economics. London 2000.

(3) Spangenberg, J. H.: Die ökonomische Nachhaltigkeit der Wirtschaft. Berlin 2005.

(4) Vgl. auch den Beitrag Grosskurth, J.: Sinn und Unsinn von Nachhaltigkeitsindikatoren. In: Ökologisches Wirtschaften $1 / 2004$

\section{Der Autor}

Jochachim Spangenberg ist wissenschaftlicher Mitarbeiter am Sustainable Europe Research Institute (SERI).

Kontakt: Grosse Telegraphenstr. 1 D, 50676 Köln. Tel. 0221-2168-70, E-Mail: JoachSpangenberg@aol.com 
(c) 20I0 Authors; licensee IÖW and oekom verlag. This is an article distributed under the terms of the Creative Commons Attribution Non-Commercial No Derivates License (http://creativecommons.org/licenses/by-nc-nd/3.o/), which permits unrestricted use, distribution, and reproduction in any medium, provided the original work is properly cited. 